\title{
Atomic force microscope base nanolithography for reproducible micro and nanofabrication
}

\begin{abstract}
Atomic force microscopy nanolithography (AFM) is a strong fabrication method for micro and nano structure due to its high spatial resolution and positioning abilities. Mixing AFM nanolithography with advantage of silicon-on-insulator (SOI) technology provides the opportunity to achieve more reliable Si nanostructures. In this letter, we try to investigate the reproducibility of AFM base nanolithography for fabrication of the micro/nano structures. In this matter local anodic oxidation (LAO) procedure applied to pattern a silicon nanostructure on p-type (1015 cm-3) SOI using AFM base nanolithography. Then chemical etching is applied, as potassium hydroxide (saturated with isopropyl alcohol) and hydrofluoric etching for removing of Si and oxide layer, respectively. All parameters contributed in fabrication process were optimized and the final results revealed a good potential for using AFM base nanolithography in order to get a reproducible method of fabrication.
\end{abstract}

Keyword: Local anodic oxidation; Silicon-on-insulator; Nanolithography; Atomic force microscope 